\title{
Structural Model Reveals Key Interactions in the Assembly of the Pregnane X Receptor/Corepressor Complex
}

\author{
Ching Y. Wang, Chia W. Li, J. Don Chen and William J. Welsh
}

Department of Pharmacology, University of Medicine \& Dentistry of New Jersey (UMDNJ), Robert Wood Johnson Medical School and UMDNJ Informatics Institute, Piscataway, NJ 08854

W.J.W (Corresponding Author) Department of Pharmacology, University of Medicine \&

Dentistry of New Jersey, Robert Wood Johnson Medical School, 675 Hoes Lane, Piscataway,

New Jersey, 08854; Email: welshwj@umdnj.edu; Tel: 732-235-3234. 


\section{Running Title: Molecular Dynamics of PXR-SMRT Interactions}

\section{Corresponding Author:}

William J. Welsh, Ph.D. Department of Pharmacology, University of Medicine \& Dentistry of New Jersey, Robert Wood Johnson Medical School, 675 Hoes Lane, Piscataway, New Jersey, 08854; Email: welshwj@umdnj.edu; Tel: 732-235-3234; Fax: 732-235-3475.

Number of text pages: 19; Figure: 4; References: 27.

Number of words in Abstract: 197, Introduction: 477; Results and Discussion: 957.

ABBREVIATIONS: NR, nuclear receptor; PXR, pregnane receptor; SXR, steroid and xenobiotic receptor; SMRT, silencing mediator for retinoid and thyroid hormone receptor; ID1, nuclear receptor interacting domain 1; ID2, nuclear receptor interacting domain 2; $\alpha \mathrm{AF}$, activation function 2; hRAR, human retinoic acid receptor; LBD, ligand binding domain; MD, molecular dynamics; EM, energy minimization; RMSD, root mean square deviation; PDB, Protein Data Bank. 
MOLPHARM/2006/022368

\begin{abstract}
The human pregnane $\mathrm{X}$ receptor (PXR), also known as steroid and xenobiotic receptor (SXR), is a member of the orphan nuclear receptors and mediates the mammalian xenobiotic response with broad specificity and implications for drug clearance. The mouse pregnane $\mathrm{X}$ receptor is highly similar to the human ortholog in structure but with subtle species differentiation in the ligand binding domain (LBD). The C-terminal helix named $\alpha \mathrm{AF}$ or AF-2 helix in other nuclear receptors is responsible for transcription activation by recruiting co-activators through conformational change. In the absence of ligands, PXR can also repress gene expression by interacting with transcriptional corepressors such as the silencing mediator for retinoid and thyroid hormone receptor (SMRT). We first constructed homology models of completed LBD with two SMRT nuclear receptor (NR)-interacting domains (ID1 and ID2) respectively. We then performed energy minimization and molecular dynamics simulations on these systems to study the specific interactions between the interacting domains and LBD. Further experimental results supported and validated the observed preference of SMRT toward ID2 over ID1. Our modeling results revealed the key interactions that account for the binding preference. Here, we propose structural models of the PXR-LBD/SMRT-ID1 and PXR-LBD/SMRT-ID2 complexes to understand their molecular interactions and potential inhibitory mechanism.
\end{abstract}


Molecular Pharmacology Fast Forward. Published on February 1, 2006 as DOI: 10.1124/mol.106.022368

This article has not been copyedited and formatted. The final version may differ from this version.

MOLPHARM/2006/022368

\section{Introduction}

The human pregnane $\mathrm{X}$ receptor (PXR), also known as the steroid and xenobiotic receptor $(\mathrm{SXR})$ is an essential regulator of genes encoding several major types of cytochrome P450 enzymes and transporters (e.g. multidrug resistance-1, MDR1). PXR is crucial because of its involvement in the regulation of phase I (cytochrome P450), phase II (conjugating), and phase III (ABC family transporters) metabolizing and detoxifying enzymes, coordinately regulating steroid, drug, and xenobiotic clearance in the liver and intestine (Blumberg et al., 1998; Kliewer et al., 1998; Xu et al., 2002). It is activated by compounds that regulate the gene expression of the enzymes and transporters, and therefore contributes significantly to drug-drug interactions. PXR binds to response elements and initiates transactivation (Wilson and Kliewer, 2002).

PXR belongs to a superfamily of the nuclear receptors (NR) and regulates gene transcription in a ligand-dependent manner. In contrast to most other nuclear receptors, PXR possesses a large ligand-binding pocket that is spherical in shape, extremely hydrophobic, and expandable. These features render PXR a promiscuous nuclear receptor that is capable of binding a wide variety of structurally diverse ligands (Watkins et al., 2001). However, like other members of the NR superfamily, the PXR LBD is multifunctional, capable of ligand binding, dimerization, transcription activation, and interactions with transcriptional cofactors. The Cterminal helix termed AF-2 helix or $\alpha \mathrm{AF}$ is responsible for transcription activation by recruiting co-activators through conformational rearrangement (Leo and Chen, 2000). PXR can also repress gene expression by interaction with transcriptional corepressors such as the nuclear receptor corepressor (N-CoR) (Horlein et al., 1995) and the silencing mediator for retinoid and thyroid hormone receptor (SMRT) in the absence of ligands (Chen and Evans, 1995; Ordentlich et al., 1999; Park et al., 1999). 
Molecular Pharmacology Fast Forward. Published on February 1, 2006 as DOI: 10.1124/mol.106.022368

This article has not been copyedited and formatted. The final version may differ from this version.

MOLPHARM/2006/022368

SMRT is a general corepressor for many nuclear receptors, such as thyroid receptor and peroxisome proliferators-activated receptor $\alpha$ (PPAR $\alpha)$. It has two nuclear receptor (NR)interacting domains, named ID1 and ID2 (Li et al., 1997). Each ID has a signature, CoRNR box or LxxxIxxxI/L motif, a corepressor motif responsible for interactions with NRs (Hu and Lazar, 1999; Nagy et al., 1999; Perissi et al., 1999). The two IDs can interact with a single NR or with a NR dimer, respectively, and different NRs have different binding preferences. In a recent report, Johnson et al.(Johnson et al., 2006) have demonstrated that PXR binds preferably to ID2 over ID1. Interpretation of the molecular modeling results in this report provide clues to the key residues involved in the binding preference.

In order to understand the key interactions that account for the preference, we have employed computational approaches to model and simulate the PXR LBD and SMRT NRinteracting domains. Since there are only two known antagonists, ET-743 (Synold et al., 2001) and certain polychlorinated biphenlys (PCB's) (Tabb et al., 2004), elucidation of inhibitory mechanism through the recruitment of SMRT by PXR is a crucial step to advance our understanding of drug-drug interaction and potential cancer drug therapeutics. Here, we propose structural models of the PXR-LBD/SMRT-ID1 and PXR-LBD/SMRT-ID2 complexes to elucidate their molecular interactions and potential inhibitory mechanism. 
Molecular Pharmacology Fast Forward. Published on February 1, 2006 as DOI: 10.1124/mol.106.022368

This article has not been copyedited and formatted. The final version may differ from this version.

MOLPHARM/2006/022368

\section{Materials and Methods}

All calculations were conducted on SGI Octane R12000 machines. Development of the PXR-SMRT structural models were carried out in three steps: homology modeling and energy minimization (EM) and molecular dynamics (MD) simulations. The structural models were further validated by experimental results. The procedures employed for each step are described below.

\section{Homology Modeling}

Numerous X-ray crystal structures of PXR are available for use as a template (RCSBPDB accession number: 1ILG, 1ILH, 1M13, 1NRL, 1SKX) to build our homology models; however, none of them contain an intact LBD. In particular, residues 178-209 form a highly flexible loop which is unresolved in these crystal structures. This loop is purported to be involved in PXR's promiscuity with respect to the diversity of its ligands. The crystal structure of PXR in apo-form (PDB ID = 1ILG) was chosen to be the initial template; as this structure represents the most suitable conformation of PXR LBD in complex with its corepressor. The flexible loop of 15 amino residues, although not resolved in the x-ray crystal structure, was generated by using Loopy module of Jackal (http://trantor.bioc.columbia.edu/programs/jackal), a protein structure modeling package. The final model for PXR/SMRT-ID2 complex was built from the x-ray crystal structure of SMRT bound to PPAR $\alpha$ and GW6471 (PDB ID = 1KKQ) (Xu et al., 2002), and the intact LBD of PXR. The PPAR $\alpha /$ SMRT-ID2 structure was first aligned and superimposed onto the PXR-LBD structure to minimize the root-mean-square deviation (RMSD) of backbone atoms in the core of the LBD using Sybyl6.9 (http://www.tripos.com) (Sybyl6.9). This superimposition minimized the RMSD between the core PXR helices and the corresponding 
Molecular Pharmacology Fast Forward. Published on February 1, 2006 as DOI: 10.1124/mol.106.022368

This article has not been copyedited and formatted. The final version may differ from this version.

MOLPHARM/2006/022368

PPAR $\alpha$ helices, and effectively superimposed their co-repressor binding sites. The coordinates and amino acids of SMRT-ID2 peptide were transferred from the PPAR $\alpha$ structure to the PXRLBD, and then examined visually. A few residue side chains of SMRT were rotated to improve its fit with the $\alpha \mathrm{AF}$ helix of PXR using the program INSIGHT-II (http://www.accelrys.com) . The final PXR/SMRT-ID2 structural model was then subjected to further EM and MD simulations. For the PXR/SMRT-ID1 complex, we mutated the ID2 sequence to the corresponding ID1 sequence while preserving the remaining structural components. EM and MD simulations were employed for each construct in order to explore protein-protein interactions in the binary complex (PXR-LBD/SMRT) under normal physiological conditions. Two structural models were generated and refined: PXR-LBD/SMRT-ID2 and PXR-LBD/SMRT-ID1 (Figure 1(b), 1(c)).

\section{Energy Minimization (EM) and Molecular Dynamics (MD) Simulations}

Refinement of the PXR/SMRT-ID1 and -ID2 structural models was conducted by EM and MD calculations using AMBER7 (Case et al.). After EM, PXR-LBD in complex with SMRT-ID1 was solvated by a $9 \AA$ radius shell of TIP3 water molecules (Ryckaert et al., 1977). The solvated system was energy minimized in two steps: first, 1000 iterations of constrained steepest descent (SD) whereby only the water molecules were free to move to eliminate improper steric interactions; second, 5000 interactions of SD and 5000 iterations of conjugated gradient (CG) minimization was conducted on the entire system. MD simulations were then performed on the resulting system using the standard force-field parameter set parm99 in AMBER7 with dielectric constant $\varepsilon=1$ and cutoff distance $=9.0 \AA$ applied for both electrostatic and vander Waals interactions. The SHAKE algorithm (Ryckaert et al., 1977) was implemented for bonds 
Molecular Pharmacology Fast Forward. Published on February 1, 2006 as DOI: 10.1124/mol.106.022368

This article has not been copyedited and formatted. The final version may differ from this version.

MOLPHARM/2006/022368

involving hydrogen atoms and the time step $=2 \mathrm{fs}$. The system was then coupled to a Berendsen bath at $300 \mathrm{~K}$ by using a coupling constant $\tau_{\mathrm{T}}=2$ ps (Berendsen et al., 1984). The system was gradually increased in temperature from 100 to $300 \mathrm{~K}$ over 10 ps of simulation time with the volume held constant (ensemble NVT). When the system approached $\mathrm{T} \cong 300 \mathrm{~K}$ and the density $\cong$ $1.0 \mathrm{~g} / \mathrm{ml}$, constant pressure and temperature controls (NPT) were applied to the simulation was conducted for $1 \mathrm{~ns}$. The pressure of the system was raised to 1 atmosphere (ensemble NPT). The same force-field parameters were then employed for EM and MD simulations of the PXR/SMRT-ID2 system using the same protocol as for PXR/SMRT-ID1. Structures retrieved from the equilibrated (post-500 ps) system were averaged and energy minimized using 1000 iterations of SD and 5000 iterations of CG. The final refined structures were analyzed with PROCHECK (Laskowski et al., 1993; Morris et al., 1992).

\section{Experimental Validation Using GST-pulldown Assay}

The human pregnane X receptor (hPXR, GenBank accession no. NR1I2), human retinoid acid receptor-alpha (hRAR $\alpha, \mathrm{NR} 1 \mathrm{~B} 1)$, and human thyroid receptor-beta (hTR $\beta$, NR1A2) were created by standard molecular cloning techniques, including restriction digestion with endonucleases (New England BioLabs), and PCR using Pfu polymerase (Stratagene), followed by end-joining ligation with T4 DNA ligase (Roche Molecular Biochemicals). Derivatives of SMRT (Chen and Evans, 1995; Park et al., 1999) were created similarly. All constructs used for in vitro transcription/translation were in the pCMX vector (Umesono et al., 1991). Glutathione Stransferase (GST) fusion protein purification was conducted as described (Frangioni and Neel, 1993) with glutathione-conjugated agarose beads (Sigma). GST pulldown assay was conducted as described (Ghosh et al., 2002). 
Molecular Pharmacology Fast Forward. Published on February 1, 2006 as DOI: 10.1124/mol.106.022368

This article has not been copyedited and formatted. The final version may differ from this version.

MOLPHARM/2006/022368

\section{Results and Discussion}

To elucidate the recruitment of corepressors by PXR, we first compared the sequences of the $\alpha$ AF helix of PXR, SMRT-ID1, SMRT-ID2, and the AF-2 of human retinoid acid (RARAF2) (Fig. 1(a)). We observed a similarity between the PXR $\alpha$ AF helix and the corepressor LxxIxxxI/L motif. In contrast, the RAR AF-2 helix is more related to the coactivator LxxLL motif (Fig. 1(a)). In general, a nuclear receptor recruits a coactivator or corepressor through a combination of hydrophobic interactions by the canonical motifs and other protein-protein interactions by the "charged clamps" and/or hydrogen bonds. The sequence similarity of the PXR $\alpha$ AF helix with the corepressor motif is further supported by the fact that the helix contains a two-turn $\alpha$-helix, similar to the proposed structure of the corepressor motif. The primary amino acid sequences and the secondary structure of the helix support the ability of the $\alpha \mathrm{AF}$ helix to mediate or inhibit PXR binding to SMRT.

To further assess the ability of PXR to recruit SMRT, we first constructed homology models of PXR with various the SMRT nuclear receptor (NR)-interacting domains (ID1 and ID2). We subsequently performed minimization (EM) and molecular dynamics (MD) simulations to study the specific interactions between the two IDs and the PXR LBD. We further validated the structural models and the binding of ID1 and ID2 to PXR using GST pull-down assays.

Numerous x-ray crystal structures for use as a structural template are not intact and lack of a highly flexible loop of residues 178-209, which are unresolved in these crystal structures. The crystal structure of PXR in apo-form (PDB ID = 1ILG) was chosen to be the initial template. This unresolved loop of 15 amino residues was then generated by using Loopy module of Jackal (http://trantor.bioc.columbia.edu/programs/jackal), a protein structure modeling package. The 
Molecular Pharmacology Fast Forward. Published on February 1, 2006 as DOI: 10.1124/mol.106.022368

This article has not been copyedited and formatted. The final version may differ from this version.

MOLPHARM/2006/022368

structural model for the PXR-LBD/SMRT-ID2 complex was built from the x-ray crystal structure of SMRT bound to PPAR and GW6471 (PDB ID = 1KKQ), and the x-ray crystal structure of the apo-form PXR (PDB ID = 1ILG). For the PXR-LBD/SMRT-ID1 complex, we mutated the ID2 sequence to ID1 sequence and kept the rest of structure complex intact. EM and MD simulations were employed for each construct. Two structural models were generated and refined: PXR-LBD/SMRT-ID1, and PXR-LBD/SMRT-ID2.

MD simulations on the PXR-LBD/SMRT-ID1 structure confirm the stability of 3D structure and the equilibrium of binary complex, as evidenced by the rapid convergence of the root mean square deviation (RMSD) of the $\alpha$-carbon backbone of the sampled conformation compared with the initial structure (Figure 2, straight line). Similarly, RMSD analysis of the MD trajectory (Figure 2, dashed line) of the PXR-LBD/SMRT-ID2 structural model indicated a stable 3D structure. PROCHECK assessment also displayed acceptable quality, aside from a few amino acid residues falling outside the "allowed" regions associated with the flexible loop mentioned above (residues 178-209).

Based on the assumption that the corepressor site overlaps with the coactivator site, SMRT-ID2 and the PXR LBD were shown to form three key interactions: one charge clamp and two salt bridges (Figure 3(b)). The essential charge clamp typically formed by Lys 259 of helix 3 (Watkins et al., 2003) and coactivator was conserved for both ID1 and ID2 domains of SMRT. Specifically, Lys259 formed a charge clamp with Ile2143 of ID1 and Leu2350 of ID2, respectively (Figure 3). Another essential charge clamp typically formed by Glu427 of the $\alpha \mathrm{AF}$ helix (Watkins et al., 2003) was not found in either domain. The preference of SMRT for ID2 over ID1 can be attributed to two additional key interactions: Glu427 of $\alpha$ AF with Lys2348 of ID2, and Glu270 of helix 4 with Arg2347 of ID2 (Figure 3(b)). These two additional interactions 
Molecular Pharmacology Fast Forward. Published on February 1, 2006 as DOI: 10.1124/mol.106.022368

This article has not been copyedited and formatted. The final version may differ from this version.

MOLPHARM/2006/022368

are salt bridges and, as such, are a stronger than a standard hydrogen bond. Our structural model suggested that Pro423 and Lys2348 of ID2 formed another hydrogen bond; however, this interaction is presumably much weaker than the two salt bridges.

Another possible reason for reduced preference of SMRT for ID1 over ID2 is due to its slightly unfavorable spatial environment inside the cofactor pocket. Specifically, the ID1 fragment encounters steric hindrance with the $\alpha \mathrm{AF}$ helix. Furthermore, ID1is anchored with the $\alpha \mathrm{AF}$ helix through only two hydrogen bonds: one between Glu427 of $\alpha \mathrm{AF}$ and Gln2137 of ID1, and the other between Pro423 of $\alpha$ AF and His2129 of ID1 (Fig. 3(a)).

The preceding discussion underscores the difference in ascribing the differential binding among ID1 and ID2 to a solitary factor. Indeed, ID1 and ID2 bear great similarity in their primary amino acid sequence and secondary structure, and both share the canonical corepressor motif. Our computational approaches enabled us to elucidate putative key interactions that account for the observed binding difference in binding of these two similar interacting domains to SMRT. The differences in the type and number of nonbonded interactions between the two domains of SMRT and PXR LBD revealed by our structural analysis may explain the specific preference for the recruitment of SMRT-ID2 by PXR.

The results from the MD studies are in accordance with the experimental results. Biological experiment evidence using GST-pull down assay showed the differential preferences of three nuclear receptors toward individual SMRT domains (Figure 4). Human RAR $\alpha$ in general binds preferably to ID1, while human TR $\beta$ and human PXR bind preferably to SMRT-ID2. Further biological studies, including immunofluorescence colocalization and luciferase reporter assays, are detailed in the recent publication by Johnson et al. (Johnson et al., 2006) Taken together, our computational and experimental results provide new insights into the assembly of 
Molecular Pharmacology Fast Forward. Published on February 1, 2006 as DOI: 10.1124/mol.106.022368

This article has not been copyedited and formatted. The final version may differ from this version.

MOLPHARM/2006/022368

the PXR-LBD/SMRT-ID2 complex by proposing the key interactions that account for the binding preferences and possible molecular mechanism of $\alpha \mathrm{AF}$ helix-mediated inhibition of SMRT binding. In future work, we will perform molecular simulations and biological experiments to assess the binding of PXR- $\alpha \mathrm{AF}$ to NR-domains (ID1 and ID2) of SMRT. These future studies will complement ongoing experimental mutational analysis of the specific residues (e.g., P423 and E427) identified in the $\alpha$ AF helix to validate the present modeling observations.

\section{Conclusions}

Three-dimensional structural models of PXR/SMRT complexes were constructed using computational homology modeling techniques and refined by EM and MD simulations. The refined structural models were further evaluated by the biological assay using GST-pulldown. Visual analysis of PXR/SMRT NR-interacting domains identified specific residues (viz. Lys259, Glu270, Pro423, and Glu427 of PXR LBD, and Arg2347, Lys2348, and Leu2350 of SMRT-ID2) that may play an important role in corepressor binding affinity and preference.

Here, we have provided a potential molecular model of interactions that determine the assembly of the PXR/SMRT-ID1 and -ID2 complexes. Our modeling results reveal the structural basis for the binding preference of SMRT-ID2 over SMRT-ID1 to the PXR $\alpha$ AF helix. Since there are very few known antagonists of PXR, the selectivity of NR-interacting domains can be further employed to regulate the gene expression mediated by PXR. These studies provide insights into the molecular interactions between PXR and SMRT corepressor, which may have important implications for understanding the role of co-repressors in regulating the manifold biological activities of PXR. 
Molecular Pharmacology Fast Forward. Published on February 1, 2006 as DOI: 10.1124/mol.106.022368

This article has not been copyedited and formatted. The final version may differ from this version.

MOLPHARM/2006/022368

\section{Acknowledgments}

We thank the Informatics Institute of UMDNJ for computer time. The PXR-LBD/SMRT-

ID1 and -ID2 models are available upon request to the authors (welshwj@umdnj.edu). 
Molecular Pharmacology Fast Forward. Published on February 1, 2006 as DOI: 10.1124/mol.106.022368

This article has not been copyedited and formatted. The final version may differ from this version.

MOLPHARM/2006/022368

\section{References}

InsightII 2000: Accelrys Inc., San Diego, CA.

Berendsen HJC, Postm JPM, van Gunsteren WF, Di Nola A and Haak JR (1984) Molecular dynamics with coupling to an external bath. J. Chem. Phys. 81:3684-3690.

Blumberg B, Sabbagh W, Juguilon H, Bolado JJ, van Meter CM, Ong ES and Evans RM (1998) SXR, a novel steroid and xenobiotic sensing nuclear receptor. Genes and Development 12:3195-3205.

Case DA, Pearlman JW, Caldwell TE, Cheatham III J, Wang WS, Ross CL, Simmerling TA, Darden KM, Merz RV, Stanton AL, Cheng JJ, Vincent M, Crowley V, Tsui H, Gohlke RJ, Radmer Y, Duan J, Pitera I, Massova GL, Seibel UC, Singh PK, Weiner PA and Kollman PA (2002) AMBER7, University of California, San Francisco.

Chen JD and Evans RM (1995) A transcriptional co-repressor that interacts with nuclear hormone receptors. Nature 377:454-457.

Frangioni JV and Neel BG (1993) Solubilization and Purification of Enzymatically Active Glutathione S-Transferase (pGEX) Fusion Proteins. Analytical Biochemistry 210:179187.

Ghosh JC, Yang X, Zhang A, Lambert MH, Li H, Xu HE and Chen JD (2002) Interactions that determine the assembly of a retinoid $\mathrm{X}$ receptor/corepressor complex. PNAS 99:58425847.

Horlein AJ, Naar AM, Heinzel T, Torchia J, Gloss B, Kurokawa R, Ryan A, Kamei Y, Soderstrom M, Glass CK and Rosenfeld MG (1995) Ligand-independent repression by the thyroid hormone receptor mediated by a nuclear receptor co-repressor. Nature 377:397-404.

Hu X and Lazar MA (1999) The CoRNR motif controls the recruitment of corepressors by nuclear hormone receptors. Nature 402:93-96.

Johnson DR, Li C-W, Chen L-Y, Ghosh JC and Chen JD (2006) Regulation and Binding of Pregnane X Receptor by Nuclear Receptor Corepressor Silencing Mediator of Retinoid and Thyroid Hormone Receptors (SMRT). Mol Pharmacol 69:99-108.

Kliewer SA, Moore JT, Wade L, Staudinger JL, Watson MA, Jones SA, McKee DD, Oliver BB, Willson TM and Zetterstrom RH (1998) An Orphan Nuclear Receptor Activated by Pregnanes Defines a Novel Steroid Signaling Pathway. Cell 92:73-82.

Laskowski RA, MacArthur MW, Moss DS and Thornton JM (1993) PROCHECK: a program to check the stereochemical quality of protein structures. J. Appl. Cryst. 26:283-291.

Leo C and Chen JD (2000) The SRC family of nuclear receptor coactivators. Gene 245:1-11.

Li H, Leo C, Schroen DJ and Chen JD (1997) Characterization of Receptor Interaction and Transcriptional Repression by the Corepressor SMRT. Mol Endocrinol 11:2025-2037.

Morris AL, MacArthur MW, Hutchinson EG and Thornton JM (1992) Stereochemical quality of protein structure coordinates. Proteins 12:345-364.

Nagy L, Kao H-Y, Love JD, Li C, Banayo E, Gooch JT, Krishna V, Chatterjee K, Evans RM and Schwabe JWR (1999) Mechanism of corepressor binding and release from nuclear hormone receptors. Genes Dev. 13:3209-3216.

Ordentlich P, Downes M, Xie W, Genin A, Spinner NB and Evans RM (1999) Unique forms of human and mouse nuclear receptor corepressor SMRT. Proc. Natl. Acad. Sci. USA 96:2639-2644. 
MOLPHARM/2006/022368

Park EJ, Schroen DJ, Yang M, Li H, Li L and Chen JD (1999) SMRTe, a silencing mediator for retinoid and thyroid hormone receptors-extended isoform that is more related to the nuclear receptor corepressor. Proc. Natl. Acad. Sci. USA 96:2639-2644.

Perissi V, Staszewski LM, McInerney EM, Kurokawa R, Krones A, Rose DW, Lambert MH, Milburn MV, Glass CK and Rosenfeld MG (1999) Molecular determinants of nuclear receptor-corepressor interaction. Genes Dev. 13:3198-3208.

Ryckaert JP, Ciccotti G and Berendsen HJC (1977) Numerical integration of Cartesian equation of motion of a system with constraints: molecular dynamics of $\mathrm{N}$-alkanes. J. Comput. Phys. 23:327-341.

Syby16.9 Tripos, Inc., St. Louis, MO.

Synold TW, Dussault I and Forman BM (2001) The Orphan Nuclear Receptor SXR Coordinately Regulates Drug Metabolism and Efflux. Natural Medicine 7:584-590.

Tabb MM, V. K, Grun F, Zhou C, Welsh WJ and Blumberg B (2004) Highly Chlorinated PCBs Inhibit the Human Xenobiotic Response Mediated by the Steroid and Xenobiotic Receptor (SXR). Environmental Health Perspectives 112:163-169.

Umesono K, Murakami KK, Thompson CC and Evans RM (1991) Direct repeats as selective response elements for the thyroid hormone, retinoic acid, and vitamin D3 receptors. Cell 65:1255-1266.

Watkins RE, Davis-Searles PR, Lambert MH and Redinbo MR (2003) Coactivator Binding Promotes the Specific Interaction Between Ligand and the Pregnane X Receptor. Journal of Molecular Biology 331:815-828.

Watkins RE, Wisely GB, Moore LB, Collins JL, Lambert MH, Williams SP, Wilson TM, Kliewer SA and Redinbo MR (2001) The human nuclear xenobiotic receptor PXR: Structural determinants of directed promiscuity. Science 292:2329-2333.

Wilson TM and Kliewer SA (2002) PXR, CAR and metabolism. Nat Rev Drug Discov 1:259266.

Xu HE, Stanley TB, Montana VG, Lambert MH, Shearer BG, Cobb JE, McKee DD, Galardi CM, Plunket KD, Nolte RT, Parks DJ, Moore JT, Kliewer SA, Willson TM and Stimmel JB (2002) Structural basis for antagonist-mediated recruitment of nuclear co-repressors by PPAR[alpha]. Nature 415:813-817. 
MOLPHARM/2006/022368

\title{
Footnotes
}

Funding for this research was provided by New Jersey Commission on High Education, the Informatics Institute of the University of Medicine and Dentistry of New Jersey. Funding from the US Environmental Protection Agency (USEPA \#TBN-13753), National Risk Management Research Laboratory, is gratefully acknowledged.

\author{
Dr. William J. Welsh \\ Department of Pharmacology \\ University of Medicine \& Dentistry of New Jersey-Robert Wood Johnson Medical School \\ 675 Hoes Lane \\ Piscataway, NJ 08854 \\ Email: welshwj@umdnj.edu
}


MOLPHARM/2006/022368

\section{Figure Legends}

Figure1. (a) Sequence Comparison of the PXR $\alpha$ AF helix with SMRT-ID1 and SMRT-ID2. The C-terminal sequence alignment is based on the co-activator and co-repressor motifs. (b) Structural model of PXR LBD in complex with SMRT ID2. The SMRT-ID2 helix is colored in purple, while the remaining PXR LBD is depicted as a ribbon. Individual colors represent both helices and loops. (c) Structure model of PXR LBD in complex with SMRT ID1. SMRT-ID1 peptide was generated by mutating residues of ID2 peptide to match the amino acid residues of ID1. SMRT-ID1 helix is represented as a red-colored ribbon.

Figure 2. RMSD $(\AA)$ of carbon backbone of PXR/SMRT complex is plotted versus MD simulation time. The $\alpha$-carbon backbone RMSD of PXR/SMRT-ID1 (straight line) and PXR/SMRT-ID2 (dashed line) as determined from their respective starting conformations obtained during MD simulations.

Figure 3. Binding orientation of SMRT-ID1 and-ID2 in the co-repressor site of PXR-LBD. SMRT NR-interacting domains are shown in blue as ribbons. The residues involved in proteinprotein interactions are rendered in ball-and-stick mode and colored by atom-type. Yellow dashed lines represent charge clamps, and green dashed lines represent either hydrogen bonds or salt bridges for PXR-LBD/SMRT-ID1 (A), and PXR-LBD/SMRT-ID2 (B).

Figure 4. GST-pulldown assay showed the differential preferences of three nuclear receptors: RAR $\alpha$ binds to ID1, while human TR $\beta$ and human PXR (hPXR) bind preferably to ID2. Coomassie blue stained gel ensured equal amount of protein loading. 
(a)

SXR- $\alpha$ AF 417 I HPFA TPLM QELEG I T G S

SMRT-ID1 GHQRVVTLAQHISEVTT QDYTRH SMRT-ID2 RAR-AF2 HASTN MGLEE A I $K M E I P G S M P$ RKALLMGGGKA LQ EMLLEN SEGLD AF-2 helix

(b)
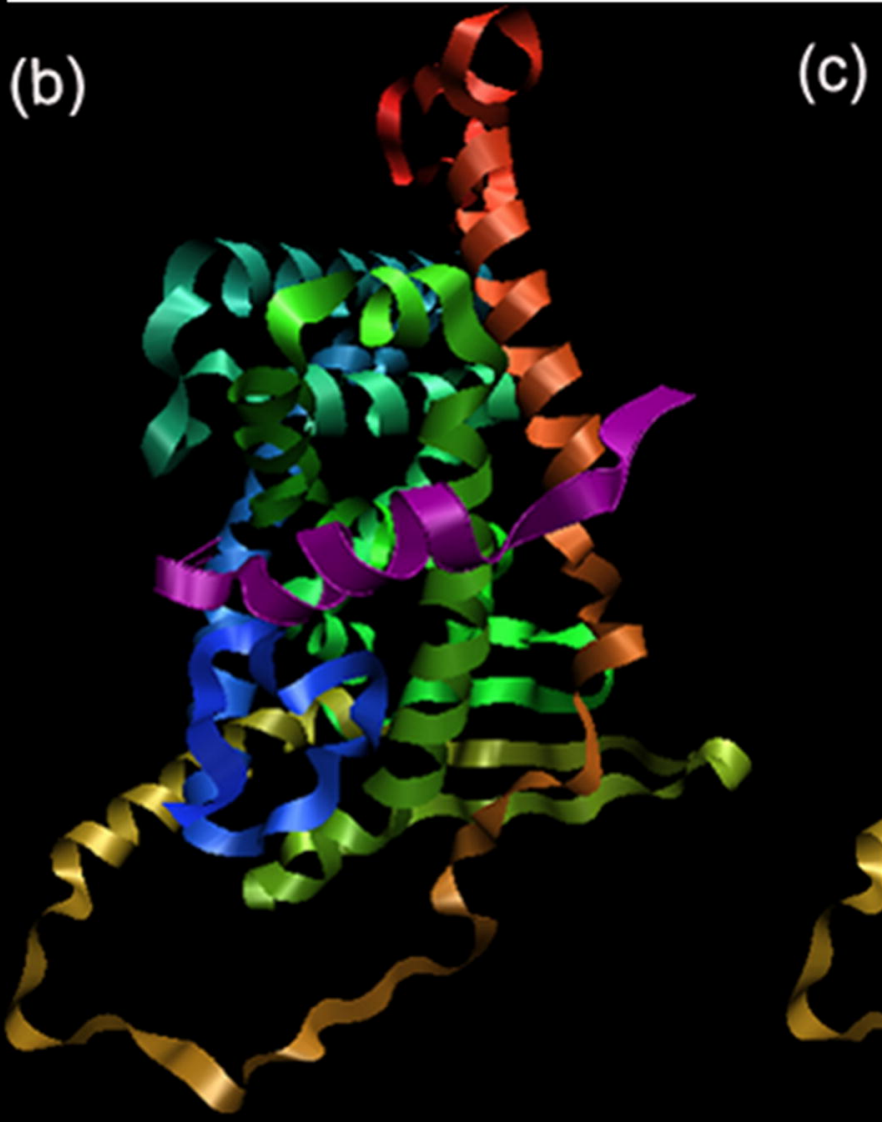

(c)

Figure1 
Figure 2

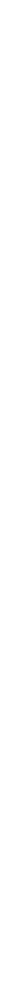


Figure3

$12143-2 \times 10$

ID1

Q2137

(a)

E427

H2129

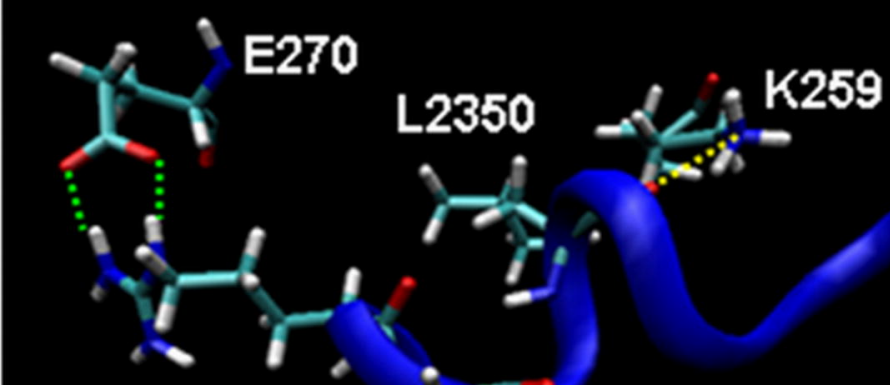

ID2

R2347

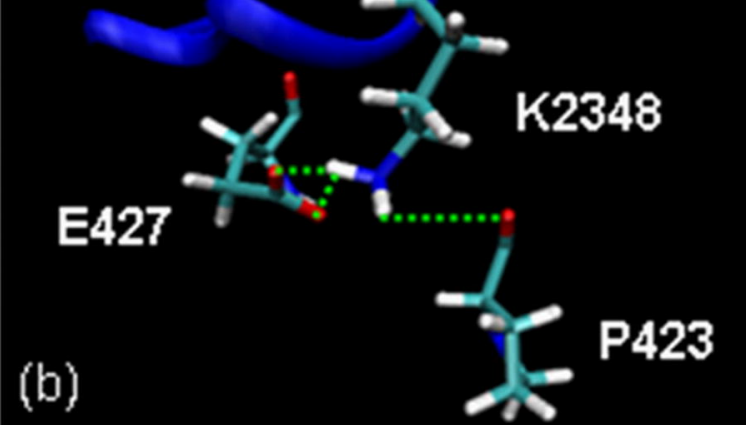


Figure 4

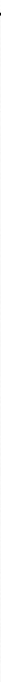

